\title{
Regulatory Guidelines for New Drug Development
}

\author{
D. Adukondalu ${ }^{1 *}$, Rajesh ${ }^{1}$, Shaik Thaslim ${ }^{1}$, E. Soumya ${ }^{1}$, M. Chandana ${ }^{1}$, G. Yamini ${ }^{1}$ \\ ${ }^{1}$ Vaagdevi College of Pharmacy, Warangal, Telangana, India.
}

*Corresponding Author: D. Adukondalu, M. Pharm, PhD, Vaagdevi College of Pharmacy, Warangal, Telangana, India.

Received date: August 09, 2021; Accepted date: August 26, 2021; Published date: September 08, 2021

Citation: D. Adukondalu, Rajesh, Shaik Thaslim, E. Soumya, M. Chandana, G. Yamini (2021) Regulatory Guidelines for New Drug Development J. Pharmaceutics and Pharmacology Research 4(3); DOI: 10.31579/2693-7247/046

Copyright: (c) 2021, D. Adukondalu, This is an open access article distributed under the Creative Commons Attribution License, which permits unrestricted use, distribution, and reproduction in any medium, provided the original work is properly cited.
Abstract investigational new drug and development of new drug
Objectives: The objective of current project include
1. Need of a new drug to investigate
2. New drug development targets
3. Understanding the properties of new dug
4. Required protocols for submission of new drug to regulatory authority
5. Regulatory requirements to get approval of new drug.

Aim: The aim of present project work is to understand the guidelines and regulatory requirements for

Keywords: drug development, regulatory authority, IND regulation, pharmacokinetic

\section{Introduction}

\section{Definition of an Investigational Product}

ICH GCP defines an investigational product as, "A pharmaceutical form of an active ingredient or placebo being tested or used as a reference in a clinical trial" (ICH GCP 1.33). This may include a marketed product that is being used in a different form than the one it was approved for, or a marketed product being used for an unapproved or new indication.

\section{Definition of an Investigational New Drug}

The Code of Federal Regulations (CFR) defines an investigational new drug as: "...a new drug or biological drug that is used in a clinical investigation." In the U.S. Food and Drug Administration (FDA) regulations, an investigational new drug is any substance (such as a drug, vaccine or other biological product) for which FDA approval is being sought. A drug may be considered "new" even if it has been in use for years if a change is proposed in its use, formulation, route of administration, use in patient population where risk would be increased, or packaging. For example, years ago the FDA approved a drug to treat high blood pressure.

Drug discovery is a process, which aims at identifying a compound therapeutically useful in treating and curing a disease. Typically a drug discovery effort addresses a biological target that has been shown to play a role in the development of the disease or starts from a molecule with interesting biological activities. The process of drug discovery involves the identification of candidates, synthesis, characterization, screening, and assays for therapeutic efficacy. Once a compound has shown its value in these tests, it will begin the process of drug development prior to clinical trials. Drug discovery and development is an expensive process due to the high costs of R\&D and human clinical tests. The average total cost per drug development varies from US\$ 897 million to US\$ 1.9 billion. The typical development time is $10-15$ years.
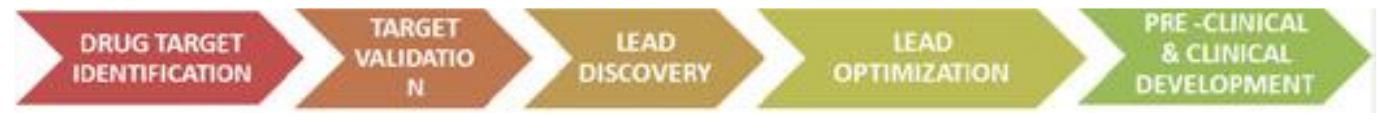

\section{Figure1: Drug Discovery Pipeline.}

The developing world suffers the major burden of infectious disease, yet the range of drugs available for the treatment of many infectious diseases is limited. In the past most drugs have been discovered either by identifying the active ingredient from traditional remedies or by serendipitous discovery [1]. At present a new approach is being tried to understand how disease and infection are controlled at the molecular and physiological level and to target specific entities based on this knowledge.

Steps in Modern Drug Discovery 


\section{Step 1: Target identification}

Target identification is the first key stage in the drug discovery pipeline. Generally speaking, a drug target is the specific binding site of a drug in vivo through which the drug exerts its action [2]. A specific drug target might have the following characteristics:

1. The drug target is a biomolecule(s), normally a protein that could exist in isolated or complex modality.

2. The biomolecules have special sites that match other.

3. The biomolecular structure might change when the biomolecule binds to small molecules and the changes in structure normally are reversible.

4. Following the change in the biomolecule's structure various physiological responses occur and induce regulation of the cell, organ, tissue, or body status.

5. The physiological responses triggered by the changes in biomolecule structure play a major role in complex regulation and have a therapeutic effect on pathological conditions.

6. The expression, activity, and structure of the biomolecule might change over the duration of the pathological process.

7. Small molecules binding to the biomolecules are drugs. As is apparent from the above discussion, a drug target is a key molecule involved in a particular metabolic or signal transduction pathway that is specific to a disease condition or a specific disease. However, the term 'drug target' itself has several limitations and is debated within the pharmaceutical industry. In this respect, several points should be kept in mind.

First, a drug target is a relative concept. For starters, a drug target is, just like other biomolecules, also a biomolecule involved in a transduction pathway. The difference between the two is only in their location and role in the transduction pathway. Another aspect is that a drug target is disease-dependent, that is, every target is involved in a special spectrum of diseases.

Second, most human diseases are rather complicated and involve many risk factors, so there are clearly many different drug targets with respect to a specific disease. Targeting a specific target could not conceivably cure a kind of disease. However, the involvement of many targets in a disease does not mean that each target shares equally in the pathogenesis of the disease and thus drugs targeting these targets would not be equally effective in the therapy of the disease.

Third, drug targets can change, which means that with the development of insights into biomolecules and their role in the pathogenesis of a certain disease, drug targets might be not as important as or may be much more important than currently believed. In fact, the establishment of drug targets is based on understanding of the pathogenesis of the disease.

Fourth, there are many drugs targeting the same target and one drug may have more than one target. The relationship between a drug and its target is not one-to- one but one-to-many or many-toone.

Fifth, when a new drug target is discovered and validated, researchers usually hope to obtain more specific drugs targeting thetarget. However, a key understanding to keep in mind is that the body is a subtle organism and a more specific drug might disrupt the homeostasis of the body. Compared to aspirin, rofecoxib is a specific COX-2 inhibitor. However, studies had shown that rofecoxib increases cardiovascular risks, resulting in rofecoxib's withdrawal from the drug market.

Sixth, a drug target usually refers to a single biomolecule. According to whether there are drugs available, a drug target can be classified into two classes: established drug targets and potential drug targets. The former are those for which there is a good scientific understanding, supported by a lengthy publication history regarding both how the target functions in normal physiology and how it is involved in human pathology.
Furthermore, there are many drugs targeting this target. The latter are those biomolecules whose functions are not fully understood and which lack drugs targeting them. Potential targets suggest directions for completely new drug research.

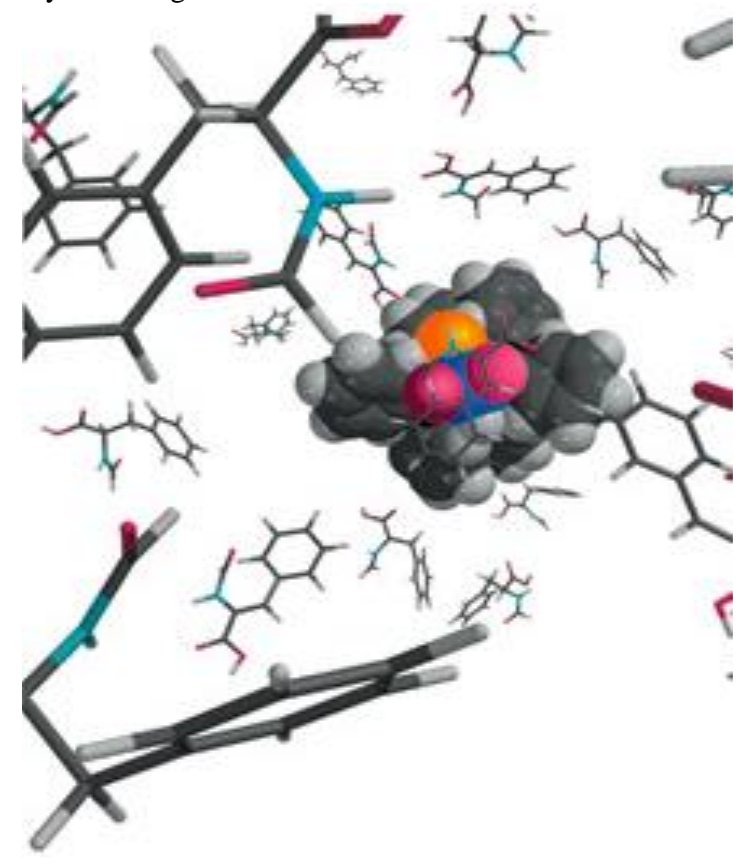

Figure 2: Lead molecule.

\section{Step 2: Target validation}

New target validation is the basis of completely new drug exploration and the initial step of drug discovery. New drug target validation might be of great help not only to new drug research and development but also provide more insight into the pathogenesis of target related diseases [3]. Basically, the target validation process might include six steps:

1. Discovering a biomolecule of interest.

2. Evaluating its potential as a target.

3. Designing a bioassay to measure biological activity.

4. Constructing a high-throughput screen.

\section{Performing screening to find hits.}

\section{Evaluating the hits.}

The drug discovery process starts with the identification or growing evidence of, biological targets that are believed to be connected to a particular condition or pathology. Information supporting the role of these targets in disease modulation can come from a variety of sources [4]. Traditionally, the targets have been researched and largely discovered in academic laboratories, and to a lesser extent in the laboratories of pharmaceutical and biotechnology companies. Basic research into understanding the fundamental, essential processes for signaling within and between cells and their perturbation in conditions has been the basic approach for establishing potential targets suitable for drug intervention.

\section{Step 3: Lead discovery}

Once a disease- associated molecular target has been identified and validated in disease models, in the lead generation phase, compounds are identified which interact intact animals or disease-related cell based models that can provide information about the integrative response of an organism to a pharmacological intervention and hereby help to predict the 
possible profile of new drugs in patients. This is accomplished primarily with knock-out or knock-in animal models; small molecule molecular target in vitro usually precedes the validation of the therapeutic concept in vivo; together this defines its clinical potential. Validation involves studies in molecular target in vitro usually proceeds with the target protein and modulate its activity. Libraries of compounds that are either synthetic chemicals, peptides, natural or engineered proteins, or antibodies are exposed to the target in a manner that will detect and isolate those members of the library that interact with and, preferably, have an effect on the target [5-8]. The compounds selected are called "leads". Initially screening can be performed by searching for compounds that bind to the target, but binding is not sufficient for therapeutic activity. More recent screening procedures include an activity-based readout as part of the initial screening assay. For example, if the goal is to inhibit a protein that is involved in activating the expression of a particular gene or set of genes, the assay can include readout to determine if the expression of the gene is reduced by the compound. Such assays can be cell-based, but more often they are enzymatic assays that can be performed in a high-throughput manner for compounds that bind to the target, but binding is not sufficient for therapeutic activity. More recent screening procedures include an activity-based readout as part of the initial screening assay. For example, if the goal is to inhibit a protein that is involved in activating the expression of a particular gene or set of genes, the assay can include readout to determine if the expression of the gene is reduced by the compound. Such assays can be cell-based, but more often they are enzymatic assays that can be performed in a high-throughput manner.

\section{Step 4: Lead optimization}

Lead optimization is a process that begins with a compound that displays an interesting biological action and ends with the identification of the best analog. Molecules are chemically modified and subsequently characterized in order to obtain compounds with suitable properties to become a drug. Leads are characterized with respect to pharmacodynamic properties such as efficacy and potency in vitro and in vivo, Physiochemical properties, pharmacokinetic properties, and toxicological aspects.

Potency - refers to the amount of drug required for its specific effect to occur.

Efficacy - measures the maximum strength of the effect itself, at saturating drug concentrations.

Pharmacokinetics - determines the fate of xenobiotics. It explains about "What the body does to the drug". It often divided into areas examining the extent and rate of adsorption, distribution, metabolism, and excretion (ADME).

Pharmacodynamics- It determines the biochemical and physiological effects of drugs, the mechanism of drug action and the relationship between drug concentration and effect. It explains about "What the drug does to the body". This process ideally requires the simultaneous optimization of multiple parameters and is thus a time consuming and costly step.

This is often the tightest bottleneck in drug discovery. However, by turning a biologically active chemical into an effective and safe drug, lead optimization contributes essentially towards added value in the drug discovery process.

\section{Step 5: Pre-clinical and clinical development}

Pre-clinical development: The pre-clinical development includes the following: develop large scale synthesis; animal safety studies; carcinogenicity tests; drug delivery; elimination and metabolism studies; drug formulation experiments; dose-ranging studies in animals. Wide ranging dosages of the compounds are introduced to the cell line or animal in order to obtain preliminary efficacy and pharmacokinetic information.

\section{Clinical Development}

The NIH organizes clinical trials into 5 different types:

1. Treatment trials: test experimental treatments or a new combination of drugs.

2. Prevention trials: look for ways to prevent a disease or prevent it from returning.

3. Diagnostic trials: find better test or procedures for diagnosing a disease.

4. Screening trials: test methods of detecting diseases.

5. Quality of life trials: explore ways to improve comfort \& quality of life for individuals with a chronic illness.

Pharmaceutical clinical trials are commonly classified into 4 phases.

Phase 0 - A recent designation for exploratory, first in human trials designed to expedite the development of promising therapeutic agents by establishing early on whether the agent behaves in human subjects as was anticipated from preclinical studies.

Phase 1 - A small group of healthy volunteers (20-80) are selected to assess the safety, tolerability, pharmacokinetics, \& pharmacodynamics of a therapy. Normally include dose ranging studies so that doses for clinical use can be set/adjusted.

Phase 2- Performed on larger groups (20-300) \& are designed to assess the activity of the therapy, \& continue phase 1 safety assessments.

Phase 3 -Randomized controlled trials on large patient groups (hundreds to thousands) aimed at being the definitive assessment of the efficacy of the new therapy, in comparison with standard therapy. Side effects are also monitored. It is typically expected that there be atleast two successful phase 3 clinical trials to obtain approval from the FDA. Once a drug has proven acceptable, the trial results are manufacturing procedures, formulation details, shelf life, etc. This document is submitted to the FDA for review.

Phase 4 - Post-launch safety monitoring \& ongoing technical support of a drug may be mandated or initiated by the pharmaceutical company designed to detect rare or long term adverse effects over a large patient population \& timescale than was possible during clinical trials.

\section{Lead Discovery Methods}

Leads are discovered in different ways. They can be found by pure serendipity, by systematic screening, by the chemical modification of known active compounds or by a rational approach. Actually these methods may be interconnected \& progress simultaneously. An outline of the four approaches is given below:

The serendipitous pathway: Hundreds of drugs that are now in the market were discovered by unexpected observations or "lucky accidents" in everyday life or in medical clinics.

The screening pathway: Random screening is an important approach in pharmaceutical research. A great number of molecules are screened randomly in the hope of finding a compound with a specific biological activity. This approach entered a new dimension with combinatorial chemistry \& high through-put screening that allows considering the chemistry \& the screening in an automated manner.

The chemical modification pathway: Using this traditional method, analogs with improved biological activity are made by minor chemical modifications of known active compounds without changing their 
chemical backbone. The rational driving the modification is the synthetic accessibility of the molecules $\&$ the desire not to modify too much of the already known active compound.

The rational pathway: Rational drug design is a process that begins with a validated biological target, exploits the structural specificity of that target \&ends with the identification of a drug candidate that optimally interacts with it $\&$ triggers the desired biological action. Genomics allows the linking of specific genes to specific diseases. Rational drug design starts with this gene information \& converts it into new drug candidates.

\section{Advanced technologies involved in drug discovery}

The following advanced technologies are involved in drug discovery process.

Genomics: The genome of the organism is the complete genetic make-up of the entire DNA complement, of that organism. Genomics, then, is the study of entire genomes.

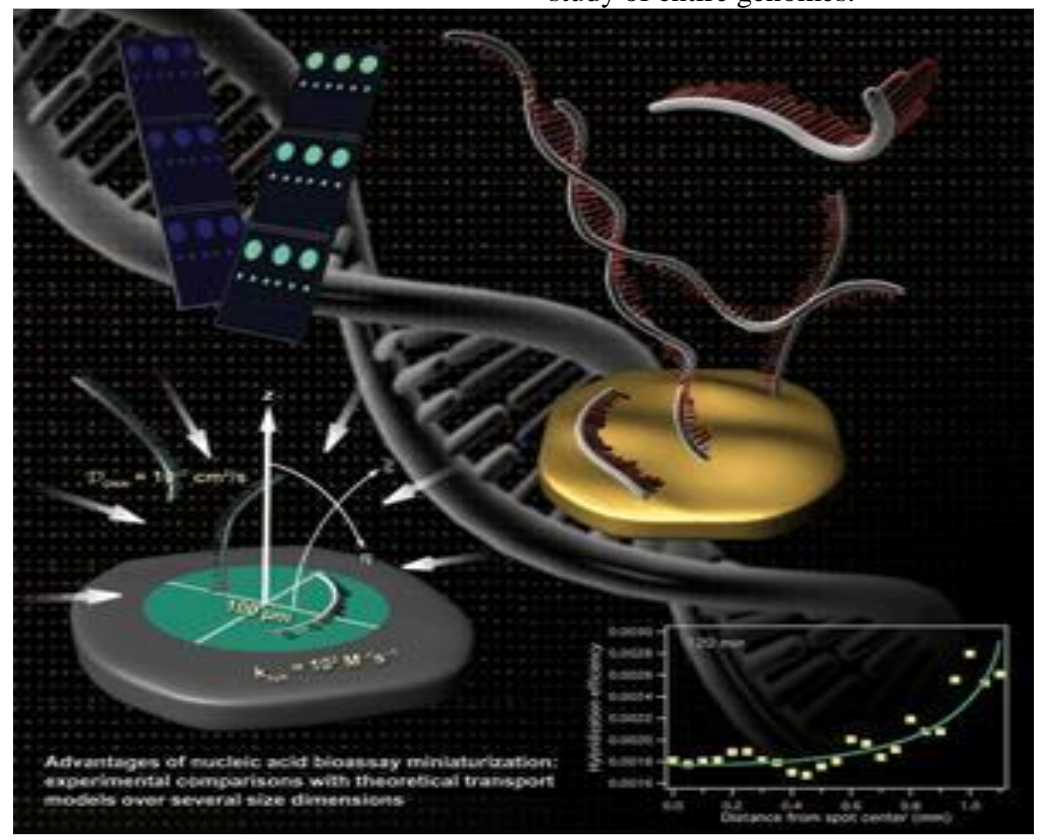

Figure 3: Genomics involved in drug discovery.

The intention of executing the sequencing and analysis of the entire human genome was to enable more rapid and effective identification of disease-associated genes and thereby provide it to drug companies with pre-validated targets. Genomic approaches have made significant advances into the discovery of functions of human genes and their involvement in diseases.
Proteomics: Proteomics is the systematic high-throughput separation and characterization of proteins within biological systems. Importantly, it is at the protein level that disease processes become manifest and at which most drugs act.

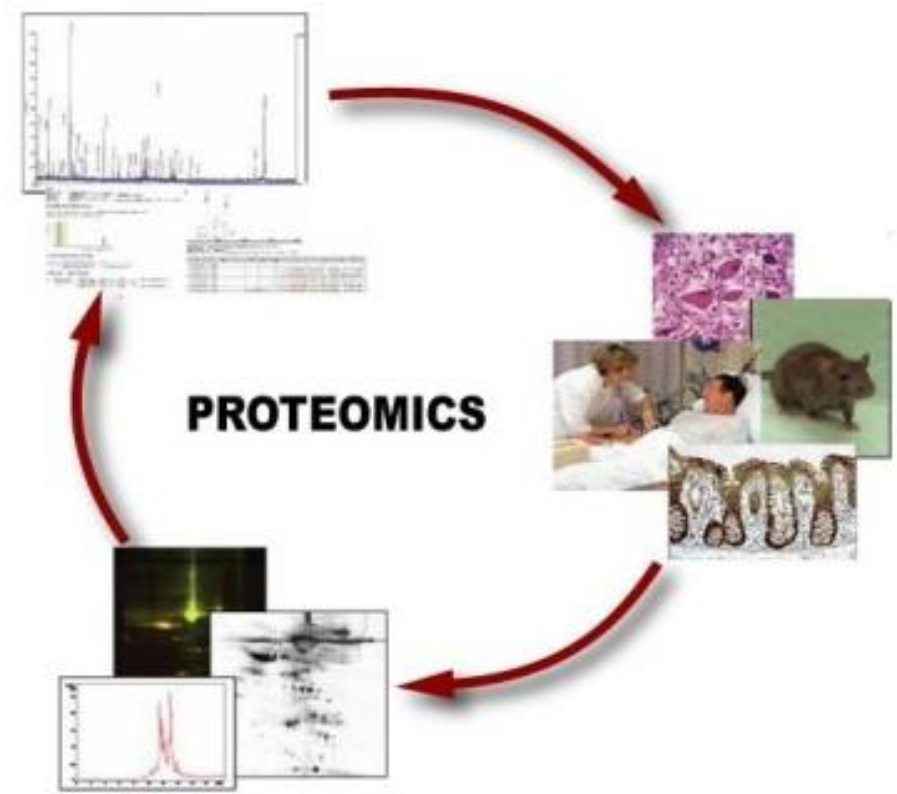




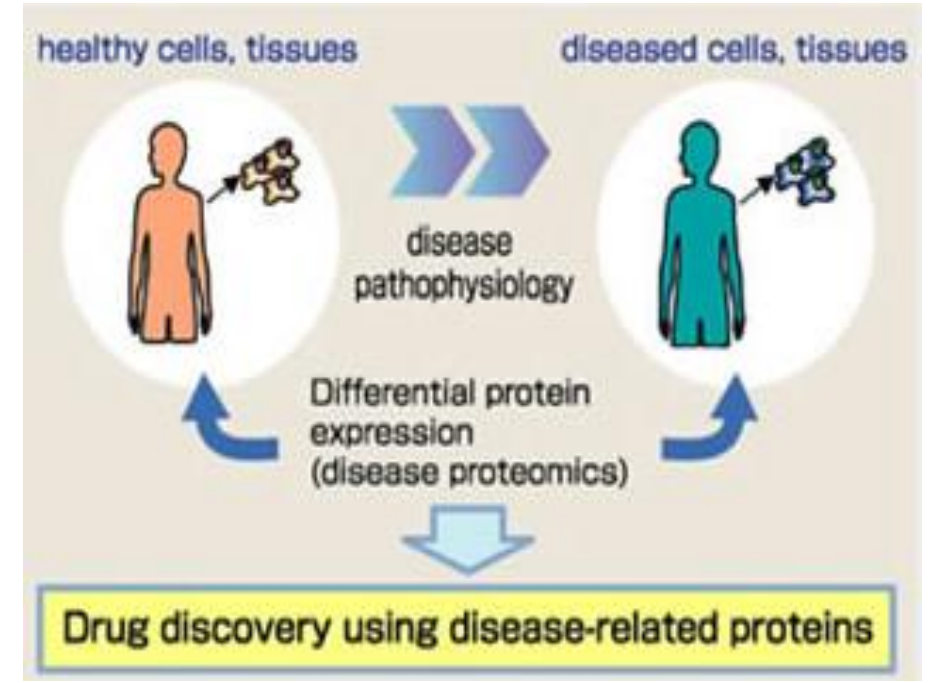

Figure 4: Proteomics involved in drug discovery.

Proteomics is consequently assuming a central place modern drug development with a wide spectrum of practical applications embracing diagnostic target discovery, target validation lead compound. Selection, investigation, modes of action, toxicology and chemical development of drug.
Bioinformatics: Bioinformatics is having profound implications for pharmaceutical and diagnostic concerns. Structural biology and bioinformatics have assisted in lead optimization and target identification where they have well established roles; they can now contribute to lead discovery, exploiting high throughput methods of structure determination that provide powerful approaches to screening of fragment binding [9].

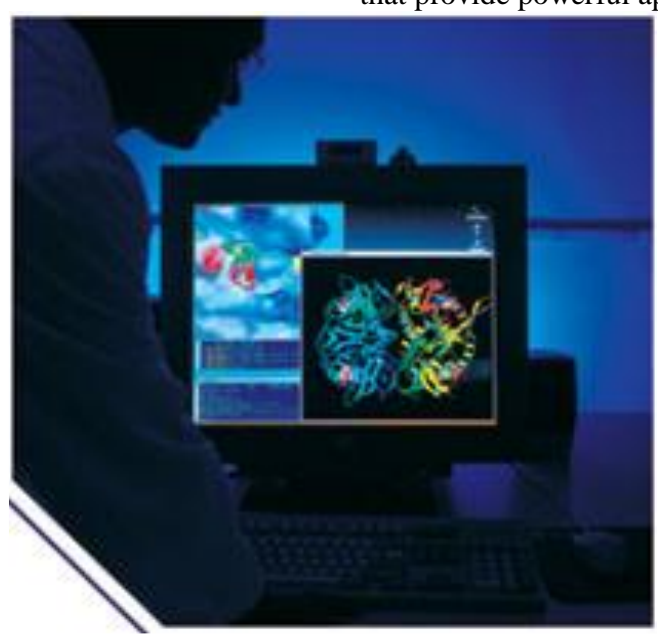

Figure 5: Bioinformatics involved in drug discovery.

Bioinformatics is being increasingly used to support drug discovery process by providing functionally predictive information mined from databases and experimental datasets using a variety of computational tools.

Combinatorial chemistry: Although many disciplines and sciences play important roles in the discovery of a drug, combinatorial chemistry plays a central role because of its ability to create and produce molecules. In addition, combinatorial chemistry is an active participant in drug development from the synthesis of substances until its bulk production. Combinatorial chemistry can create large population of analogs of a given scaffold. The analogs are synthesized in successive steps with the use of robotics. The analogs are included in "combinatorial libraries" consisting of a great number of wells, each one containing in solution several dozens of compounds. Only a few milligrams of compounds can be obtained by this method, however this is generally sufficient for primary biological screening. By producing larger and diverse compound libraries, one simply increases the probability of finding novel Compounds of interesting therapeutic value.

High-throughput Screening Technique (HTS): High-throughput screening (HTS) is an approach to drug discovery that has gained widespread popularity over the last three or four years. HTS is the process of assaying a large number of potential effectors of biological activity against targets (a biological event). 


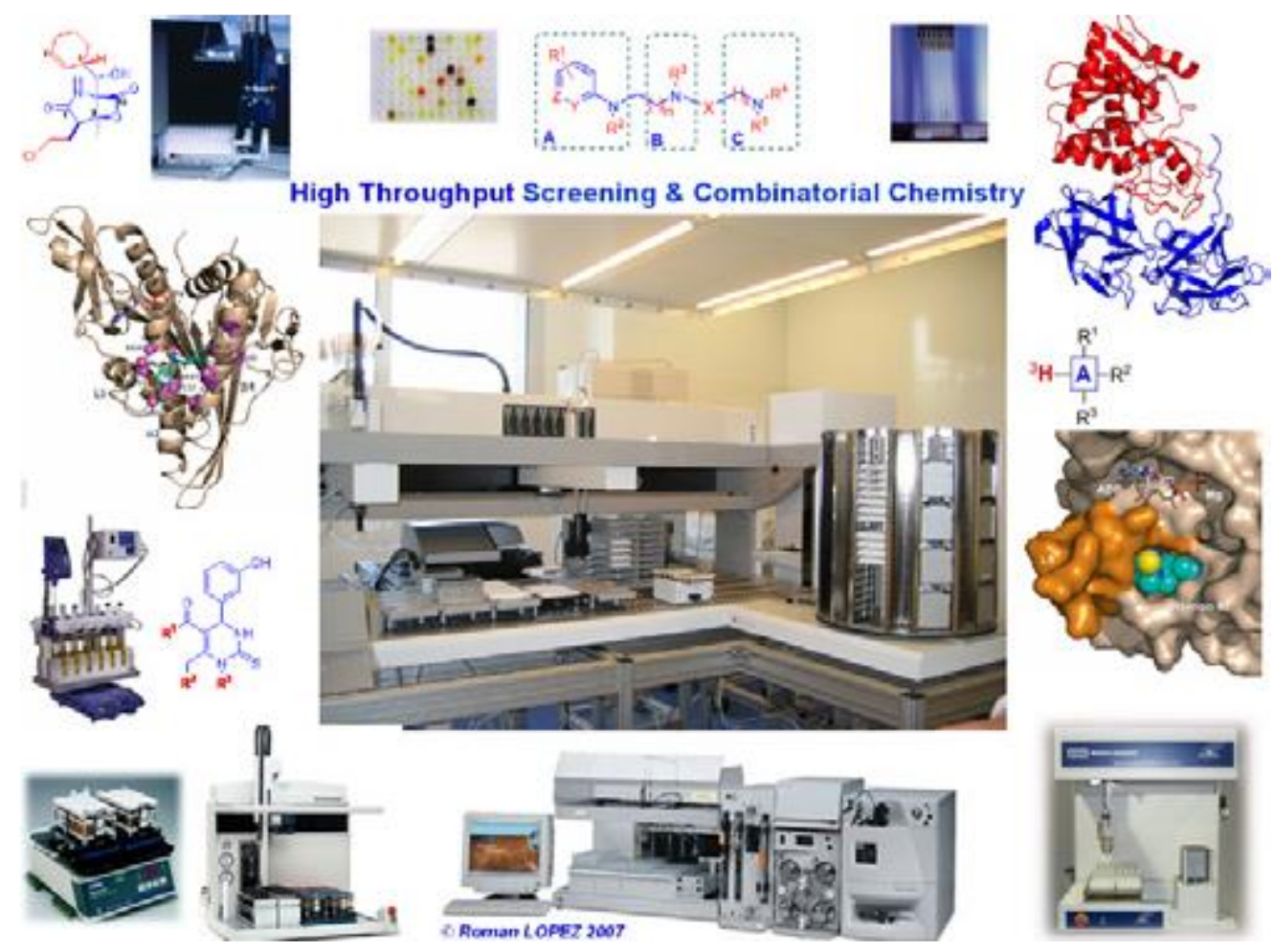

Figure 6: High Throughput Screening and Combinatorial Chemistry involved in Drug.

The methods of HTS are applied to the screening of combinatorial chemistry, genomics, protein, and peptide libraries [10]. The goal of HTS is to accelerate drug discovery by screening large libraries often composed of hundreds of thousands of compounds (drug candidates) at a rate that may exceed 20,000 compounds per week.

\section{Discovery}

Computational methods: Computational methods can be used to predict or simulate how a particular compound interacts with a given protein target. They can be used to assist in building hypothesis about desirable chemical properties when designing the drug and they can be used to refine and modify drug candidates. Virtual Screening (VS) is a general term for computational methods that use computers to screen a database of virtual drug candidates (called compounds to identify promising candidates (leads) [11]. The following three advanced computational methods are used in drug discovery process.

\section{Molecular docking}

When the structure of the target is available, usually from X-ray crystallography, the most commonly used virtual screening method is molecular docking. Molecular docking programs try to predict how a drug candidate binds to a protein target without performing a laboratory experiment. It is necessary to know the geometrical structure of both the ligand and the target protein in order to use molecular docking.

\section{Quantitative Structure-Activity Relationships (QSAR)}

QSAR is an example of a method which can be applied regardless of whether the structure is known or unknown, QSAR tries to formalize what is experimentally known about how a given protein interacts with some tested compounds [12-14]. In terms of the lock and key metaphor, we do not know what the lock looks like, but we do know which keys work, and which do not. QSAR can be considered as the method of trying to build a model for why some keys work and others do not.

Pharmacophore mapping: A pharmacophore is a set of structural features in a molecule that is recognized at a receptor site and is responsible for that molecule's biological activity. Pharmacophore Mapping is a geometrical approach. A pharmacophore can be thought of as a 3D model of characteristic features of the binding site of the investigated protein. It may describe properties like: "In region A a positive charge is needed, in region $\mathrm{B}$ a hydrogen donor, region $\mathrm{C}$ may not be occupied..." and so on. A pharmacophore can also be thought of as a template, a partial description of a molecule where certain blanks need to be filled. Pharmacophore can also be built without knowing the structure of the target. This can be 


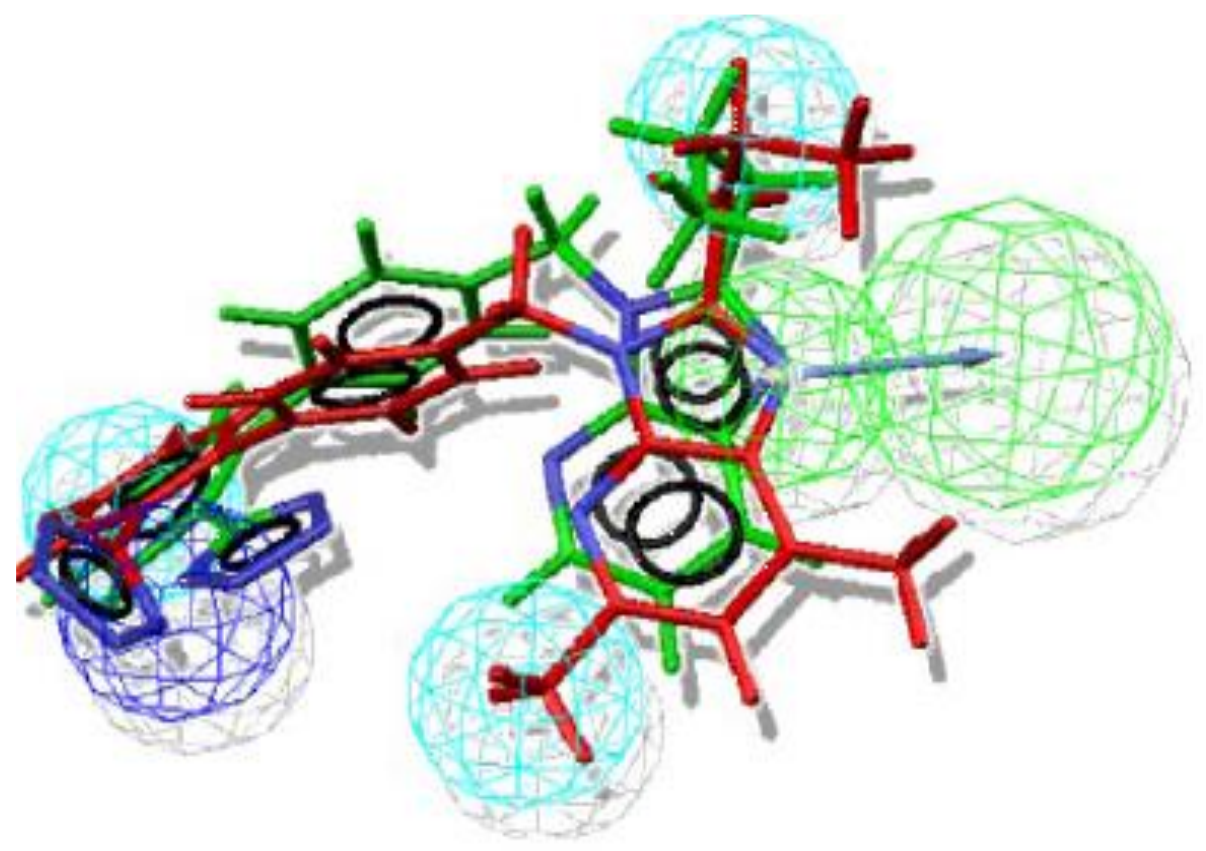

Figure 7: An example of a pharmacophore model. Spheres highlight important chemical groups that are present in both molecules (red and green sticks).

Done by extracting features from compounds which are known experimentally to interact with the target in question. Afterwards, the derived pharmacophore model can be used to search compound databases (libraries) thus screening for potential drug candidates that may be have interest.

\section{NMR-Based screening methods}

Nuclear Magnetic Resonance (NMR) techniques are widely used in the drug discovery process. The primary feature exploited in these investigations is the large difference in mass between drugs and receptors (usually proteins) and the effect that this has on the rotational or translational correlation times for drugs bound to their targets. Many NMR parameters, such as the diffusion coefficient, spin diffusion, nuclear over hauser enhancement, and transverse and longitudinal relaxation times, are strong functions of either the overall tumbling or translation of molecules in solution. This has led to the development of a wide variety of NMR techniques applicable to the elucidation of protein and nucleic acid structure in solution, the screening of drug candidates for binding to a target of choice, and the study of the conformational changes that occur in a target on drug binding.

\section{Methodology}

The manufacturer of the drug now wants to test it as a treatment for anxiety in adults. This new use of the drug would be considered investigational. In a study protocol and other documents, an investigational new drug may be referred to as the "study drug," "experimental product," "experimental drug," "new intervention," or similar term. Investigational new drugs are regulated under CFR Title 21 Part 312

Labeling of an Investigational New Drug the labeling of an investigational new drug: Must include the following statement: "Caution: New Drug Limited by Federal (or United States) law to investigational use." Must not be false or misleading and should not imply that the drug is safe or effective for the investigational purpose

Control of an Investigational New Drug An investigational new drug may be given to participants only under supervision by the principal investigator or by a sub-investigator. (Usually, the person supervising the administration of an investigational new drug is a physician.) The investigator cannot supply the investigational new drug to any person who is not authorized to receive it. Research that involves the use of controlled substances must comply with U.S. Drug Enforcement Administration regulations (21 CFR 1300-end). When studying an investigational new drug that is considered a controlled substance, the investigator must take adequate precautions to prevent theft or diversion of the $2 / 14$ drug into illegal channels of distribution. Such precautions include storing the investigational new drug "in a securely locked, substantially constructed cabinet or other securely locked, substantially constructed enclosure, access to which is limited." Promotion of and Charging for Investigational New Drugs Neither an investigator nor a sponsor may promote (that is, endorse or advertise) an investigational new drug as safe or effective for the investigational purpose. In addition: An investigational new drug cannot be distributed commercially or in a test market. An investigation cannot be prolonged "after finding that the results of the investigation appear to establish sufficient data to support a marketing application." In other words, if there is good evidence that the investigational new drug is safe and effective, the study should be stopped and no other participants enrolled. Charging for an investigational new drug in a clinical trial is not permitted without approval from the FDA unless the drug is being provided for treatment use.

\section{Investigational New Drugs Requirements}

The Investigational New Drug Application A sponsor who wishes to conduct a clinical trial that involves an investigational new drug must submit an Investigational New Drug application (IND) to the FDA. In IND studies, the IND holder is considered to be the sponsor. Information that must be provided in an IND Information that must be provided in an IND includes the following: The identity and contact information of the sponsor and the phase (or phases) of the trial. A commitment that an IRB will be responsible for initial and continuing review of the trial. The name of the drug, a list of its active ingredients, and its dosage and route of administration. The objectives and planned duration of the proposed clinical trial(s). A brief description of the plan for investigating the drug, including: The reasoning behind the drug or the study, The indication(s) 
to be studied, The kinds of clinical trials to be conducted in the first year after the IND submission, The estimated number of patients who will be given the drug in the clinical trial(s), and Any serious risks that are anticipated on the basis of animal studies or previous human studies of this drug or related drugs. For most trials, a copy of the investigator's brochure. A protocol for each planned study. (See related material summarized from The Research Protocol module.) The identities and qualifications of all investigators. (As demonstrated in a Curriculum Vitae and Form FDA 1572. Click here for instructions on completing Form FDA 1572.) The criteria for patient selection and exclusion and an estimate of the number of patients to be studied. A summary of previous experience with the drug in both animal and human studies, including (if relevant): Previous INDs, Experience with the drug in other countries, Known safety issues, chemistry and manufacturing information, and Dependence and abuse potential. When an IND Goes Into Effect

An IND is considered safe to proceed 30 calendar days after the FDA receives it unless: The FDA notifies the sponsor that the investigation described in the IND is subject to a clinical hold, or The sponsor receives written permission from the FDA to begin the study before 30 days have elapsed. At the sponsor's request, the FDA will provide advice on specific matters relating to an IND. Meetings between a sponsor and the FDA are frequently useful, and the FDA encourages such meetings to the extent that FDA resources permit. IND Exemptions for Studies of Lawfully Marketed Drugs Studies of lawfully marketed drugs are exempt from the IND regulations if they meet all five of the criteria listed in 21 CFR 312.2(b)(1). The first four of these criteria are straightforward and need no special comment. The study is not intended to support approval of a new indication or a significant change in the product's labeling. The study is not intended to support a significant change in the product's advertising. The study is conducted in compliance with Institutional Review Board (IRB) and informed consent regulations. The study will not be used to promote non-approved indications. The final criterion, however, requires interpretation. The investigation does not involve a route of administration, dosage level, use in a patient population, or other factor that significantly increases the risks (or decreases the acceptability of the risks) associated with the use of the drug. It is the investigator's responsibility to determine whether an IND is necessary for a study that involves a marketed drug. A critical question in determining whether such a study is exempt from IND regulation is whether the study "significantly increases the risk" associated with use of the drug. If an investigator is quite sure that a drug study does not require an IND, he or she can simply not submit an IND application. If the investigator is doubtful about whether an IND is required, or wishes to have proof of the IND-exempt status of a study, the IND application can be submitted with an exemption from IND guidelines requested, and FDA staff will review the application to determine whether the study is exempt. The review is limited to critical safety concerns (dose, schedule, route, and patient population). If, after this limited review, the FDA determines that a study is exempt from the requirement for an IND, it performs no further review of the application. The FDA sends a letter to the sponsor giving notice of the exemption. Prior to submission of the application, the sponsor can also set up a preIND meeting with the FDA in order to discuss any questions or concerns. IND Protocol Amendments The sponsor of an IND must submit a protocol amendment to the FDA: To describe any change in a Phase 1 protocol that significantly affects the safety of participants; or To describe any change in a Phase 2 or Phase 3 protocol that significantly affects the safety of participants, the scope of the investigation, or the scientific quality of the study. The following are examples of changes that would require the submission of a protocol amendment to the FDA: An increase in drug dosage or in the duration of participants' exposure to the drug. $7 / 14$ a significant change in the study design, such as the addition or elimination of a control group. The addition of a new test or procedure to improve monitoring for, or to reduce the risk of, an adverse event, or the dropping of a test intended to monitor safety. The protocol amendment can be implemented at study sites after the amended protocol has been submitted to and approved by the reviewing IRB, and submitted to the FDA. A protocol change that is intended to eliminate an apparent immediate hazard to participants may be implemented immediately, provided that: The FDA is subsequently notified of the change by a protocol amendment, and the reviewing IRB is also notified of the change in accordance with the IRB's rules. IND Safety Reports Sponsors must promptly review and investigate all information they receive relevant to the safety of an investigational new drug that is received from any source, foreign or domestic, including information derived from: Clinical or epidemiological studies, Animal studies, Commercial marketing experience, Reports in the scientific literature, unpublished scientific papers, and Reports from foreign regulatory authorities. The sponsor must notify the FDA of any unexpected fatal or life-threatening experience associated with the use of the drug as soon as possible but not later than 7 calendar days after the sponsor's initial receipt of the information. Sponsors must provide written notification to the FDA and to all investigators participating in a trial within 15 calendar days of any adverse event that is: Both serious and unexpected, and reasonably likely to have been caused by the investigational new drug. Subsequent, appropriate follow-up information must also be submitted, as it becomes available. The sponsor must also provide written notification of any finding from tests in laboratory animals that suggests a significant risk for human participants. The written notification must be provided as soon as possible and no later than 15 calendar days after the sponsor receives the information. IND Information Amendments and Annual Reports a sponsor must file an information amendment to report essential information about the IND that is not within the scope of a protocol amendment, IND safety report, or annual report. The following are examples of information that requires the filing of an information amendment: New information about technical features of the drug, such as its toxicology or chemistry. Discontinuation of a clinical investigation. Within 60 days of the first anniversary of the date the IND went into effect, and every subsequent year, a sponsor must submit a brief report of the progress of the investigation. This annual report must include: A brief summary of the status of each study in progress or completed. A summary of the most frequent and most serious adverse experiences. A summary of all IND safety reports submitted. A list of participants who died during participation in the investigation, with the cause of death for each participant. A list of participants who dropped out as a result of any adverse experience, whether or not the adverse experience is thought to be related to the investigational new drug. A summary of the general investigational plan for the upcoming year. An updated Investigator's Brochure, if available. A summary of any foreign market developments. A summary of any outstanding business with the FDA regarding the IND (i.e. a response to an FDA request for information). 


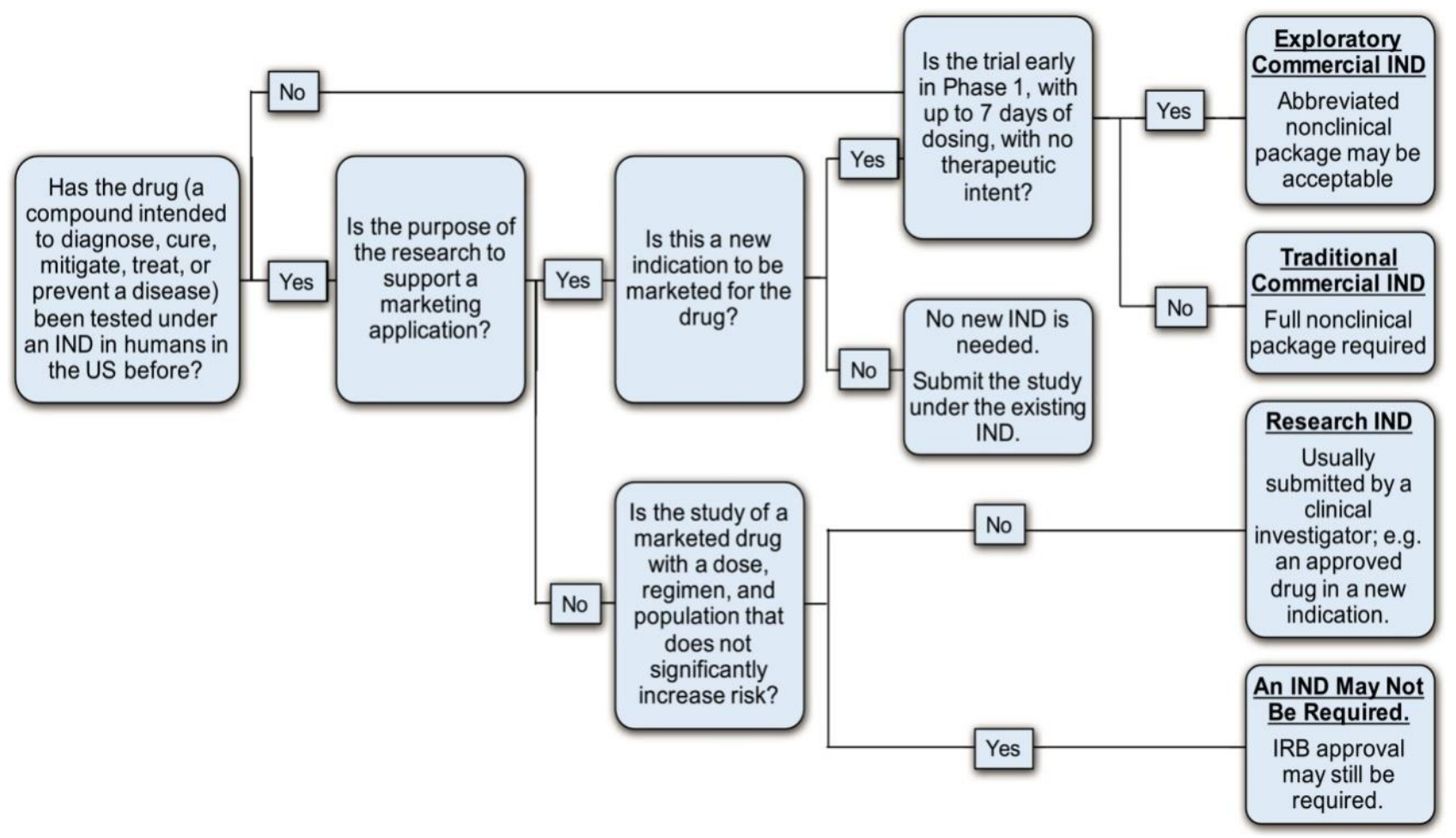

Figure 8. Process of IND

\section{Investigational New Drugs Responsibilities}

\section{Responsibilities of Sponsors}

Both sponsors and investigators who are involved in conducting a clinical trial under an IND filed with the FDA must accept and fulfill certain responsibilities. Sponsors' responsibilities include: Selecting qualified investigators. Providing investigators with the information they need to conduct the investigation. Ensuring proper monitoring of the trial. Ensuring the trial is conducted according to the plan and protocols contained in the IND. Informing the FDA and all investigators of significant new adverse effects or risks that are reasonably likely to be caused by the investigational new drug. Maintaining proper records. Disposing of unused supplies of the investigational new drug. Unless the sponsor is a sponsor-investigator, the sponsor does not actually conduct the investigation. Based on GCP guidelines, other Sponsor responsibilities include (ICH GCP E6, 5.12; 5.13; 5.14): Ensuring that the Investigational Product is manufactured in accordance with Good Manufacturing Practices. Ensuring that Investigational Product is packaged in a way that prevents contamination and unacceptable deterioration during transport and storage. Supplying investigators/institutions with the Investigational Product. Having written procedures that include instructions on the handling and storage of Investigational product that sites should follow. Maintaining sufficient quantities of the Investigational Product used in the trial to reconfirm specifications should the need arise. The above represent good examples of responsibilities the Sponsor may transfer to a Contract Research Organization (CRO), such as a clinical coordinating center. However, the ultimate responsibility for Investigational Product resides with the Sponsor. Any Investigational Product-related duties and functions that are transferred to and assumed by a CRO are specified in writing.

\section{Responsibilities of Investigators}

Investigators' responsibilities include: Providing the sponsor with a completed, signed Statement of Investigator. (Form FDA 1572. Click here for instructions on completing this form.) Conducting the trial in accordance with the signed investigator statement, protocol, and applicable regulations. Protecting the rights, safety, and welfare of trial participants. Obtaining informed consent from all trial participants. Maintaining proper records. Furnishing all required progress reports, safety reports, financial disclosure reports, and a final report. Complying with Institutional Review Board review. and Ensuring the proper handling of controlled substances. This topic is also discussed in the Roles and Responsibilities module. Based on GCP guidelines, other Investigator responsibilities include (ICH GCP E6, 4.6): Ensuring Investigational Product accountability Assigning duties for Investigational Products to a pharmacist or an appropriate individual who has the necessary license for dispensing Maintaining records of the Investigational Product from delivery at the site to dispensing to the participant as well as use by the participant, return by the participant, and reconciling all product prior to destruction. Ensuring that the Investigational Product is used in accordance with the approved protocol explaining the correct use of the Investigational Product to each participant and checking at intervals that each participant is following instructions properly. Click to view Clinical Trial Network related content CTN Why is the regulation of investigational new drugs relevant to the Clinical Trials Network? Studies performed within the NIDA Clinical Trials Network (CTN) that involve an investigational drug must be carried out in accordance with the investigational new drug regulations. The investigational new drug regulations are enacted to: Protect the safety of research participants, Ensure that participants are not exposed to experimental drugs or procedures unnecessarily, and Protect participants' rights. It is important, however, that all members of the CTN - not only those involved with studies of investigational new drugs - understand the basics of these important regulations because the reasoning behind the investigational drug regulations applies to all research involving human participants, including the International Council for Harmonization Good Clinical Practice guidelines. These principles are equally applicable to behavioral research, or to medication studies not requiring an IND, even though these studies do not involve an investigational new drug. Research participants should not be exposed to any experimental intervention unnecessarily, in 
an unsafe manner, or in a manner that fails to protect their rights. Although behavioral studies and IND-exempt medication studies conducted in the CTN are not subject to the requirement to submit IND safety reports to the FDA, CTN members who conduct such studies must submit Adverse Event/Serious Adverse Event information and reports to the DSMB Medical Monitor at NIDA. All CTN members should be familiar with the similarities and differences in terminology and reporting requirements between reports required by NIDA and those required by the FDA. Read more... Guidance Documents the FDA has incorporated the concept of Good Clinical Practice (GCP) into agency guidance documents, which are intended to help researchers comply with GCP regulations. Guidance on Good Clinical Practice may be found in the following documents: 10/14 FDA's Good Clinical Practice: Consolidated Guideline (April 1996) Clinical Investigation of Medicinal Products in the Pediatric Population Choice of Control Group and Related Issues in Clinical Trials (May 2001) Investigational New Drug Applications Prepared and Submitted by Sponsor-Investigators (2015) Although these guidance documents are not binding, they reflect the FDA's current thinking about the interpretation of the regulations. Many guidance documents are available on the FDA's Website. (Click here for a list of available guidance documents.) Guidance documents are also published in the Federal Register. Guidance documents used internationally include both the: International Conference on Harmonization Good Clinical Practice guidelines E6, and International Conference on Harmonization Good Clinical Practice guidelines E8.

\section{Phases of Clinical Trials of Investigational New Drugs}

Clinical trials of an investigational new drug are generally conducted in four phases, Phase 1 to Phase 4. Phase 0, or "exploratory" trials, also exist as small clinical trials (sometimes only a few participants) that involve dosing at a sub-therapeutic level. Phase 0 trials are not as prevalent as Phases 1-4. Each phase is designed to find out different information. Although the phases of a trial are usually conducted sequentially (one after another), they sometimes overlap. Individuals may be eligible for studies in different phases, depending on their age, general condition, the type and stage of their disease, and previous therapy, if any Phases of Clinical Trials of Investigational New Drugs

\section{PHASE 1 Trials}

Phase 1 trials are the first studies of an investigational new drug in humans. They are usually conducted in healthy volunteers. In some cases, Phase 1 trials may be conducted in individuals who have the disease the drug is intended to treat. Phase I trials generally involve between 20 and 80 participants. Phase 1 trials are designed to: Make a preliminary determination of the drug's safety in humans. Identify some of the side effects associated with the drug's use. Begin to define a safe therapeutic (healing) dose range.

\section{PHASE 2 Trials}

Phase 2 trials are usually conducted in individuals who have the disease the drug is intended to treat or are at high risk for developing the disease. Phase 2 trials are larger than Phase 1 trials but still relatively small, usually involving no more than several hundred participants. Phase 2 trials are designed to: Begin to evaluate the drug's effectiveness in treating or preventing the disease or condition of interest. Determine the optimal dosing of the drug. Determine the common short-term side effects and risks associated with the drug.

\section{PHASE 3 Trials}

Phase 3 trials are conducted after preliminary evidence from Phase 1 and 2 trials suggests that the investigational new drug is safe and effective. They usually include between several hundred and several thousand participants. Phase 3 trials are designed to: Gather additional information about the drug's safety and effectiveness to evaluate whether its benefits outweigh its risks. Compare it to other commonly used treatments for the same condition (if available) or compared to a placebo. These studies can be performed in a blinded manner. Evaluate interactions with other treatments that may be used at the same time as the investigational new drug. Provide adequate information to determine the indication for which the drug will be labeled if it is approved for marketing as well as any limitations on the drug's use that should be stated in the labeling. For example, if there were insufficient information to show that a drug can safely be given to children, the labeling would restrict the drug's use to adults.

\section{PHASE 4 Trials}

Phase 4 trials are conducted after the drug or treatment has been approved for marketing. They are designed to: Continue testing the drug or treatment to collect additional short-term safety information. Collect information about the effect of the drug or treatment in various populations. Collect information about side effects associated with longterm use of the drug.

\section{Summary}

ICH GCP refers to an Investigational Product as a pharmaceutical form of an active ingredient or placebo being tested or used as a reference in a clinical trial. In FDA regulations, an investigational new drug is any substance (such as a drug, vaccine, or biological product) for which FDA approval is being sought. A drug may be considered "new" even if it has been in use for years if a change is proposed in its use, formulation, route of administration, or packaging. A sponsor who wishes to conduct a clinical trial that involves an investigational new drug must submit an Investigational New Drug application (IND) to the FDA. Lawfully marketed drugs are exempt from the IND regulations if they meet certain criteria. Behavioral studies (like the ones conducted in the CTN) are not subject to investigational new drug regulations. Moreover, certain medication studies may be IND-exempt. It is important, nonetheless, that all researchers understand these regulations. The principle that research participants should not be exposed to experimental interventions unnecessarily, in an unsafe manner, or in a manner that fails to protect their rights is equally applicable to all studies involving human participants.

\section{References}

1. Abreu MT, Sparrow MP (2007) The role of translation and research in inflammatory bowel disease. Rev Gastroenteral Mex 72: $146-53$

2. Auffray C, Chan Z, Hood L (2009) Systems Medicine: the future of medical genomic and healthcare. Genome Med 20: 1-2.

3. Bryan NS, Bian K, Murad (2009) Discovery of the nitric oxide signaling pathway and targets for drug development. Front Biosci 14: 1-18.

4. Chen JN, Ni RZ, Xiao HB, GuoJG, Zhar JW (2009) Comparative proteomic analysis of differentially expressed proteins in human pancreatic tissue. Hepatobiliary Pancreat Dis Int 2: 193-280.

5. Fabre N, Anglade I, Vericat JA (2009) Application of toxic genomic tools in the drug research and development process. Toxicol Lett 186: 13-17.

6. Gonzalez-Sanchez JL, Serram-Rios M (2007) Molecular basic of insulin action. Drug New Perspect 20: 527-31.

7. Greinert R (2009) Skin Cancer: new markers for better prevention. Pathobiology 71: 64-81.

8. Hegen M, Keith JC Jr, Collins M, Nickerson-Nulter CL (2007) Utility of animal models for identification of potential therapeutics for rheumatoid arthritis. Ann Rheum Dis 67: 1505-15.

9. Hart WN, Hambley TW (2009) Targeted cancer therapeutics. Cancer Res 69: 1263-7. 
10. Hasko G, Linden J, Cronstein B, Pacher P (2008) Adenosine receptors: therapeutic aspects for inflammatory and immune diseases. Nat Rev Drug Discovery 9: 759-70.

11. Kochn FE (2008) High impact technologies for natural products screening. Prog Drug Res 65: 175, 177-210.

12. Matta A, Ralhan R (2009) Overview of current and future biologically based targeted therapies in head and neck squamous cell carcinoma. Head and Neck Oncol 2: 1-6.

13. Mager DE, WOO S, Jusko WJ (2009) Scaling pharamacodynamics from invitro and preclinical animal studies to humans. Drug Metab Pharmacokinet 24: 16-24.

14. McGeer EG, McGeer PL (2007) The role of anti-inflammatory agents in Parkinsons disease. CNS Drugs 21: 789-97.

15. Mullar BA (2009) Imatinib and its successors-how modern chemistry has changed drug development. Current Pharma Des 15: 120-33.

16. McHugh SM, O'Donnell J, Gillen P (2009) Genomic and Oncoproteomic advances in detection and treatment of colorectal cancer. World J Surg Oncol 1: 36.

17. McShane LM, Humberger S, Adjee AA (2009) Effective incorporation of biomarker into phase II trials. 15: 1898-905.

18. Oka M, Fagan KA, Jones PL, Mc Murtrgie (2008) Therapeutic potential of RhoA/Rho Rinase inhibitors in pulmonary hypertension. Br J Pharmacol 155: 444-54.

19. Rajcevic U, Niclou SP, Jinenez CR (2009) Proteomics strategies for target identifications and biomarker discovery in cancer. Front Biosci 14: 3292-303.

20. Rollinger JM, Langer T, Stuppner H (2006) Strategies for efficient lead structure discovery from natural products. Cur Med Chem 13: 1491-507.
21. Rollinger JM, Langer S (2008) Virtual screening for the discovery of bioactive natural products. Prog Drug Res 65: 211, 213-49.

22. Stimson L, LaThangue NB (2009) Biomarkers for predictions clinical response to HDAC inhibitors. Cancer Lett.

23. Shum BO, Rolph MS, Sewell WA (2008) Mechanisms in allergic airway inflammations - lessons from studies in the mouse. Expert Rev Mol Med 27: 10-15.

24. Taketo MM, Edelman W (2009) Mouse models of colon cancer. Gastroenterology 36: 780-98.

25. Uverdevert JB, Theodorescu D, Lee JK (2009) Utilizing the molecular gateway: the path to personalized cancer management. Clin Chem 55: 684-97.

26. Van QW, Veenstra TD (2009) How close is the bench to the bedside? Metabolic profiling in cancer research. Genome Med 1: 5.

27. Wentzensen N, Klug SJ (2009) Cervical cancer control in the era of HPV vaccination and novel biomarkers. Pathobiology 76: 829.

28. Karara AH, Edeki T, McLeod J (2010) Survey on the conduct of first-in-human clinical trials under exploratory investigational new drug applications. J Clin Pharmacol.50: 380-391. 29. DiMasi, J.A., Hansen, R.W., and Grabowski, H.G. (2003) The price of innovation: new estimates of drug development costs. Journal of Health Economics, pp. 151-185.

29. Mehta, Shreefal S. (2008) Commercializing Successful Biomedical Technologies. Cambridge: Cambridge University Press.
This work is licensed under Creative Commons Attribution 4.0 License

To Submit Your Article Click Here: Submit Manuscript

DOI: $10.31579 / 2693-7247 / 046$
Ready to submit your research? Choose Auctores and benefit from:

> fast, convenient online submission

> rigorous peer review by experienced research in your field

$>$ rapid publication on acceptance

$>$ authors retain copyrights

$>$ unique DOI for all articles

> immediate, unrestricted online access

At Auctores, research is always in progress.

Learn more auctoresonline.org/journals/pharmaceutics-andpharmacology-research 